\title{
Fault Diagnosis for Actuators in a Class of Nonlinear Systems Based on an Adaptive Fault Detection Observer
}

\author{
Runxia Guo, ${ }^{1}$ Kai Guo, ${ }^{1}$ Quan Gan, ${ }^{1}$ Junwei Zhang, ${ }^{1}$ Jiankang Dong, ${ }^{1}$ and Lanping Bai ${ }^{2}$ \\ ${ }^{1}$ College of Electronics Information and Automation, Civil Aviation University of China, Tianjin 300300, China \\ ${ }^{2}$ Process Engineering Department, Freescale Semiconductor (China) Limited, Tianjin 300300, China \\ Correspondence should be addressed to Runxia Guo; rxguoblp@163.com
}

Received 13 October 2015; Revised 28 December 2015; Accepted 13 January 2016

Academic Editor: M. Chadli

Copyright (c) 2016 Runxia Guo et al. This is an open access article distributed under the Creative Commons Attribution License, which permits unrestricted use, distribution, and reproduction in any medium, provided the original work is properly cited.

\begin{abstract}
The problem of actuators' fault diagnosis is pursued for a class of nonlinear control systems that are affected by bounded measurement noise and external disturbances. A novel fault diagnosis algorithm has been proposed by combining the idea of adaptive control theory and the approach of fault detection observer. The asymptotical stability of the fault detection observer is guaranteed by setting the adaptive adjusting law of the unknown fault vector. A theoretically rigorous proof of asymptotical stability has been given. Under the condition that random measurement noise generated by the sensors of control systems and external disturbances exist simultaneously, the designed fault diagnosis algorithm is able to successfully give specific estimated values of state variables and failures rather than just giving a simple fault warning. Moreover, the proposed algorithm is very simple and concise and is easy to be applied to practical engineering. Numerical experiments are carried out to evaluate the performance of the fault diagnosis algorithm. Experimental results show that the proposed diagnostic strategy has a satisfactory estimation effect.
\end{abstract}

\section{Introduction}

With the rapid development of modern technology, the engineering and technical personnel have put forward higher requirements for the performance and reliability of equipment. Due to the complexity of equipment and harsh work environment, in order to avoid serious losses, adopting fault diagnosis technology for real-time monitoring of equipment's status is necessary. In the past few decades, there has been a rapid development in fault diagnosis technology.

Generally, the fault diagnosis methods can be divided into model-based approaches [1-3], knowledge-based approaches [4-6], and the methods based on signal processing [79]. Among these troubleshooting approaches, knowledgebased fault diagnosis methods are strongly dependent on the diagnostic systems themselves, and the diagnostic efficiency is subjective to the integrity of the information about the diagnostic systems. The fault diagnosis algorithms based on signal processing, especially filtering theory, are easy to implement in practical applications, but the first-order linear truncation in extended Kalman filters (EKF) can lead to low precision and filters' divergence. The model-based fault diagnosis approaches can make full use of the system's information for fault location, determination of the failures' type, and fault estimation to obtain higher accuracy. Therefore, the modelbased fault diagnosis technology has been widely studied by scholars $[10,11]$. The state observer technique is very important in model-based fault diagnosis, and it is able to give specific estimated values of both failures and state variables simultaneously, which is beneficial to the subsequent design of fault-tolerant control law. Consequently, fault diagnosis based on observer has been a research hotspot $[12,13]$. The representative results are analyzed as follows.

A robust fault detection observer was designed for a Takagi-Sugeno (T-S) fuzzy model with sensor faults [14]. The approach used the technique of descriptor systems by considering sensor fault as an auxiliary state variable. The design of robust fault detection observer was formulated as an $H_{-} / H$-infinity problem. A solution of the pursued problem based on nonquadratic Lyapunov functions was then given via a linear matrix inequality formulation. An example was presented to demonstrate the design conditions. The example showed that the proposed fault detection observer was effective. 
The small-amplitude oscillatory failures in the electrical flight control system of an aircraft were specifically studied [15]. A nonlinear observer-based solution to detect oscillatory failures with small amplitude at a very early stage was presented. By narrowing the detection threshold, the proposed approach could detect a fault with small amplitude and achieve early fault warning but was not able to give a specific form of the failure.

The online fault estimation question was further pursued [16]. A nonlinear aircraft model with multiple control surfaces was considered and a fault detection and isolation (FDI) algorithm was proposed for the stuck fault detection. The estimation effect was perfect; however, the proposed algorithm was based on an ideal analytical model, without considering external disturbances and measurement noise.

Robust and accurate detection of failures in the actuators of a civil aircraft is a crucial issue. Actuator failures, if not accurately detected in time, can often lead to improper maintenance and may potentially lead to structural damage or waste of money. Motivated by the actual demand, the combination of the observer approach and the adaptive control theory is applied to carry out fault diagnosis for actuators in a class of nonlinear systems. The adaptive method has been applied in the development of fault detection observer. Adaptive control is one of the effective theories of nonlinear systems' design. By selecting adaptable compensators, the adaptive controller can achieve stable control of a nonlinear system whose parameters are not accurately known or affected by disturbances $[17,18]$. The adaptive parameters' adjusting law of the unknown fault vector has been set up, guaranteeing the asymptotic stability of the observer.

The main contributions of this paper lie in three aspects. Firstly, different from the existing papers, the fault diagnosis algorithm in this paper is not based on ideal analytical models; in other words, the random measurement noise and external disturbances are taken into account simultaneously during the whole development process [19]. The designed fault diagnosis algorithm can successfully separate the measurement noise generated by the sensors, the unknown external disturbances, and the failures when they exist at the same time. Secondly, the fault diagnosis algorithm is able to give specific estimated values of both failures and state variables rather than just giving the estimated values of the state variables or a fault warning. This is very beneficial for indepth fault analysis and taking appropriate troubleshooting actions. Thirdly, the proposed algorithm is very simple and concise, which can overcome the problem of "explosion of complexity" and is easy to be applied to practical engineering. All the above three aspects of research work are not perfect in the existing literatures.

The structure of this paper is organized as follows. An analytical model of nonlinear system with measurement noise and disturbances is presented in Section 2. Section 3 identifies the diagnostic targets and constraints under which the diagnosis algorithm is developed. A design scheme of the nonlinear adaptive fault detection observer is proposed for actuators' fault diagnosis. In addition, a theoretically rigorous proof of asymptotical stability has also been given.
Experimental results are presented in Section 4. Conclusions and future works are discussed in Section 5.

\section{Nonlinear System Statement}

Consider a fault-free nonlinear system:

$$
\begin{aligned}
& \dot{x}(t)=g(x, t)+B u(t)+M d(t), \\
& y(t)=C x(t)+N n(t),
\end{aligned}
$$

where $x(t) \in \mathfrak{R}^{n}$ denotes the state vector and it can be measured directly; $y(t) \in \mathfrak{R}^{m}$ and $u(t) \in \mathfrak{R}^{r}$ denote an output vector and a control input vector, respectively; $d(t) \in \mathfrak{R}^{n}$ denotes an external disturbance vector that is applied to the system's actuator; $n(t) \in \mathfrak{R}^{n}$ denotes a random measurement noise (band-limited white noise) vector that is generated by the system's sensors; $B$ and $C$ are appropriately dimensional constant matrices; $M$ and $N$ are full rank const. matrices; $g(x, t)$ is a nonlinear function of the state variables $x(t)$.

Remark 1. Nonlinear control system (1) describes the influence of disturbances on the state variables and the effect of measurement noise on the output, respectively.

When there are actuators' failures, the fault system can be expressed as

$$
\begin{aligned}
& \dot{x}(t)=g(x, t)+B[u(t)+f(t)]+M d(t), \\
& y(t)=C x(t)+N n(t),
\end{aligned}
$$

where $f(t) \in \mathfrak{R}^{r}$ denotes an unknown time-varying fault vector.

\section{Design of the Observer and Adaptive Fault Estimation Algorithm}

3.1. Constraint Conditions. Before designing the fault diagnosis algorithm, some constraints need to be satisfied for fault system (2).

(i) $g(x, t)$ in (1) and (2) satisfies the Lipschitz condition about state vector $x(t)$; namely, there exists constant $\delta_{1}>0$, such that

$$
\left\|g\left(x_{1}\right)-g\left(x_{2}\right)\right\| \leq \delta_{1}\left\|x_{1}-x_{2}\right\| .
$$

Remark 2. To simplify writing, $x(t)$ is abbreviated to $x$. This kind of writing method is suitable for the subsequent content.

(ii) The unknown external time-varying disturbance vector $d(t)$ is bounded; that is,

$$
\|M d\| \leq \mu_{0}
$$

where $\mu_{0} \geq 0$ represents the maximum acceptable disturbance.

The requirement that the power of external disturbances is bounded is obviously reasonable. Because the disturbances must exist actually in the running process of nonlinear system 
(1) or (2), in other words, if $\|M d\| \rightarrow+\infty$, state variables $x(t)$ and time-varying failures $f(t)$ will be "drowned" by disturbances. In this case, state estimation or fault estimation is impossible.

(iii) The sensor's measurement noise vector $n(t)$ is bounded; that is,

$$
\|N n\| \leq \mu_{1},
$$

where $\mu_{1} \geq 0$ represents the maximum amplitude of the noise.

In general, measurement noise is mingled in the feedback signal coming from the sensors, which means that the noise signal exists for real. So the measurement noise should be bounded.

(iv) The time-varying fault vector $f$ is a bounded vector with finite rate of change; that is,

$$
\begin{aligned}
& \|f\| \leq \mu_{2}, \\
& \|\dot{f}\| \leq \mu_{3},
\end{aligned}
$$

where $\mu_{2} \geq 0$ and $\mu_{3} \geq 0$ represent the maximum amplitude of the failures and the rate of change, respectively.

Constraint condition (6) has specified the applicable scope of the proposed algorithm in this paper; in other words, only bounded failures with finite rate of change can be given specific estimated values. If $\|f\| \rightarrow+\infty$ or $\|\dot{f}\| \rightarrow+\infty$, specific estimated values of failures will not be obtained.

(v) The state vector $x$ is also bounded with finite rate of change; that is,

$$
\begin{gathered}
\|x\| \leq \mu_{4}, \\
\|\dot{x}\| \leq \mu_{5},
\end{gathered}
$$

where $\mu_{4} \geq 0$ and $\mu_{5} \geq 0$ represent the maximum amplitude of the states and the rate of change, respectively.

Just as constraint condition (6), because the proposed algorithm will also give the specific estimated values of state variables, their values should also be bounded.

3.2. Fault Detection Observer. For fault system (2), the following observer is designed:

$$
\begin{aligned}
& \dot{\hat{x}}=g(\hat{x})+B[u+\hat{f}]+H(y-\hat{y}), \\
& \hat{y}=C \hat{x},
\end{aligned}
$$

where $\widehat{x} \in \mathfrak{R}^{n}, \widehat{f} \in \mathfrak{R}^{r}$, and $\widehat{y} \in \mathfrak{R}^{m}$ denote state estimation, fault estimation, and output estimation, respectively; $H$ is a finite gain matrix of the designed observer.

Here, the deviation signal $\tilde{x} \in \Re^{n}$ is defined in the following form:

$$
\tilde{x}=x-\widehat{x} .
$$

Remark 3. $x$ denotes the real values of state variables in fault system (2), which is measureable. However, $\widehat{x}$ comes from fault detection observer (8). When failures occur in actuators, namely, $\|f\| \neq 0, x$ is not equal to $\widehat{x}$; namely, $\|\widetilde{x}\| \neq 0$. In other words, $\tilde{x}$ always contains the deviation information caused by actuators' failure $f$. If there are no failures, $\widehat{x}$ asymptotically approaches $x$ and in this case, fault detection observer (8) becomes a standard state observer. So, (8) is named as "fault detection observer."

By fault system (2) and fault detection observer (8), we can obtain

$$
\begin{aligned}
\dot{\tilde{x}} & =\dot{x}-\dot{\hat{x}} \\
& =-H C \tilde{x}+(g-\widehat{g})+B(f-\widehat{f})+E d-H N n,
\end{aligned}
$$

where $\hat{g}$ denotes $g(\widehat{x})$.

3.3. Adaptive Fault Estimation. Consider the system to be supervised given by (2); the proposed adaptive fault estimation algorithm for this fault system is given by

$$
\dot{\hat{f}}=\Pi B^{T} W \tilde{x},
$$

where $\Pi=\operatorname{diag}\left\{\Pi_{11}, \Pi_{22}, \ldots, \Pi_{r r}\right\} \forall \Pi_{i i}>0, i \in[1, r]$, denotes a convergence factor matrix, namely, an adaptive rate, which can influence the estimation performance; $W$ is a symmetric positive definite matrix and the conditions that need to be satisfied for $W$ will be discussed in detail in the following.

Remark 4. Matrix $\Pi$ in (11) is discussed here. In the subsequent proof process, only the conditions for element $\Pi_{i i}$ in the diagonal of matrix $\Pi$ need to be met, that is, $\forall \Pi_{i i}>0, \forall \Pi_{i i}<$ $+\infty$, and $\forall \Pi_{i j}=0, j \in[1, r]$ and $i \neq j$. These constraint conditions can ensure that the eigenvalues of matrix $\Pi$ exist. In addition, it is easy to satisfy these constraint conditions, but the optimal value for $\Pi_{i i}$ cannot be given here.

The error signal of fault estimation $\tilde{f} \in \mathfrak{R}^{r}$ is defined in the following form:

$$
\tilde{f}=f-\widehat{f} .
$$

By constraint condition (6), it can be obtained that

$$
\dot{\tilde{f}}=\dot{f}-\dot{\hat{f}}=\dot{f}-\Pi B^{T} W \tilde{x} .
$$

Theorem 5. If $W$ in (11) and the symmetric positive definite matrix $U$ are selected to satisfy the condition

$$
(-H C)^{T} W+W(-H C)+\delta W W+\delta I=-U,
$$

where $\delta$ is a positive constant that satisfies Lipschitz condition (3) and $I$ is an appropriately dimensional unit matrix, then the fault diagnosis algorithm based on fault detection observer (8) and adaptive fault estimation algorithm (11) can ensure that state estimation error $\tilde{x}$ and fault estimation error $\tilde{f}$ are driven asymptotically to bounded constants simultaneously, in the sense that

$$
\begin{aligned}
& \lim _{t \rightarrow \infty}\|\tilde{x}(t)\| \leq \tau_{1}, \\
& \lim _{t \rightarrow \infty}\|\tilde{f}(t)\| \leq \tau_{2},
\end{aligned}
$$

where $\tau_{1}>0$ and $\tau_{2}>0$ denote two bounded positive constants. 
Proof. We now define a Lyapunov function $V(t)$ as

$$
V(t)=\tilde{x}^{T} W \tilde{x}+\tilde{f}^{T} \Pi^{-1} \tilde{f} .
$$

Remark 6. $V(t)$ evaluates deviation signal $\tilde{x}$ and fault estimation error $\widetilde{f}$ comprehensively.

After taking the time derivative of (16) and substituting (10) and (13) into it, it can be obtained that

$$
\dot{V}=\dot{\tilde{x}}^{T} W \widetilde{x}+\widetilde{x}^{T} W \dot{\tilde{x}}+\dot{\widetilde{f}}^{T} \Pi^{-1} \widetilde{f}+\widetilde{f}^{T} \Pi^{-1} \dot{\tilde{f}} .
$$

There are four terms in $\dot{V}$. In order to facilitate reading, they are expanded, respectively, into the following forms:

$$
\begin{aligned}
& \dot{\tilde{x}}^{T} W \widetilde{x}=\left[\tilde{x}^{T}(-H C)^{T}+(g-\widehat{g})^{T}+\tilde{f}^{T} B^{T}+d^{T} E^{T}\right. \\
& \left.-n^{T}(H N)^{T}\right] W \tilde{x}, \\
& \tilde{x}^{T} W \dot{\tilde{x}}=\tilde{x}^{T} W[(-H C) \tilde{x}+(g-\widehat{g})+B \tilde{f}+E d \\
& \quad-H N n], \\
& \dot{\tilde{f}}^{T} \Pi^{-1} \widetilde{f}=\left(\dot{f}-\Pi B^{T} W \tilde{x}\right)^{T} \Pi^{-1} \widetilde{f}=\dot{f}^{T} \Pi^{-1} \tilde{f} \\
& \quad-\tilde{x}^{T} W B \tilde{f}, \\
& \tilde{f}^{T} \Pi^{-1} \dot{\tilde{f}}=\tilde{f} \Pi^{-1}\left(\dot{f}-\Pi B^{T} W \tilde{x}\right)=\tilde{f}^{T} \Pi^{-1} \dot{f} \\
& \quad-\widetilde{f}^{T} B^{T} W \tilde{x} .
\end{aligned}
$$

After substituting (18) into (17), $\dot{V}$ can be expressed as

$$
\begin{aligned}
\dot{V}= & \tilde{x}^{T}\left[(-H C)^{T} W+W(-H C)\right] \tilde{x} \\
& +\left[(g-\widehat{g})^{T} W \widetilde{x}+\tilde{x}^{T} W(g-\hat{g})\right] \\
& +\left[d^{T} M^{T} W \widetilde{x}+\tilde{x}^{T} W M d\right] \\
& -\left[n^{T} N^{T} H^{T} W \widetilde{x}+\tilde{x}^{T} W H N n\right]+2 \tilde{f}^{T} \Pi^{-1} \dot{f} .
\end{aligned}
$$

After utilizing Lipschitz condition (3), the following inequality can be obtained:

$$
\begin{aligned}
\dot{V} \leq & \tilde{x}^{T}\left[(-H C)^{T} W+W(-H C)\right] \tilde{x}+2\left\|\tilde{x}^{T} W\right\| \delta\|\tilde{x}\| \\
& +2\|M d\| \lambda_{\max }(W)\|\tilde{x}\| \\
& +2\|H N n\| \lambda_{\max }(W)\|\tilde{x}\|+2 \tilde{f}^{T} \Pi^{-1} \dot{f},
\end{aligned}
$$

where $\lambda_{\max }(W)$ denotes the maximum eigenvalue of matrix $W$.

Next, the last term in (20) can be handled in accordance with the following form:

$$
\begin{aligned}
2 \tilde{f}^{T} \Pi^{-1} \dot{f} & \leq \tilde{f}^{T} \tilde{f}+\dot{f}^{T} \Pi^{-1} \Pi^{-1} \dot{f} \\
& \leq\|\widetilde{f}\|^{2}+\|\dot{f}\|^{2} \lambda_{\max }\left(\Pi^{-1} \Pi^{-1}\right),
\end{aligned}
$$

where $\lambda_{\max }\left(\Pi^{-1} \Pi^{-1}\right)$ denotes the maximum eigenvalue of matrix $\Pi^{-1} \Pi^{-1}$.

In addition, because the measurement noise vector $n(t)$ is bounded, where $\|N n\| \leq \mu_{1}$ has been declared in constraint conditions (5) and $H$ is the finite gain matrix of the designed observer, $\|H N n\|$ will also be bounded. It can be defined as follows:

$$
\|H N n\| \leq \mu_{6},
$$

where $\mu_{6}$ is a bounded positive constant.

Obviously, by combining conditions (21) and (22), (20) can be strengthened to

$$
\begin{aligned}
\dot{V} \leq & \tilde{x}^{T}\left[(-H C)^{T} W+W(-H C)\right] \tilde{x} \\
& +\delta\left[\left\|\tilde{x}^{T} W\right\|^{2}+\|\tilde{x}\|^{2}\right]+2 \mu_{0} \lambda_{\max }(W)\|\tilde{x}\| \\
& +2 \mu_{6} \lambda_{\max }(W)\|\tilde{x}\|+\|\tilde{f}\|^{2} \\
& +\|\dot{f}\|^{2} \lambda_{\max }\left(\Pi^{-1} \Pi^{-1}\right) \\
= & \tilde{x}^{T}\left[(-H C)^{T} W+W(-H C)+\delta W W+\delta I\right] \tilde{x} \\
& +2\left(\mu_{0}+\mu_{6}\right) \lambda_{\max }(W)\|\tilde{x}\|+\|\tilde{f}\|^{2} \\
& +\|\dot{f}\|^{2} \lambda_{\max }\left(\Pi^{-1} \Pi^{-1}\right),
\end{aligned}
$$

where $\mu_{0}$ has been defined in (4).

After utilizing constraint condition (6), the following inequality can be obtained easily:

$$
\|\tilde{f}\|=\|f-\hat{f}\| \leq\|f\|+\|\hat{f}\| \leq 2 \mu_{2}
$$

where $\mu_{2}$ has been defined in (6).

After substituting (6), (14), and (24) into (23), the condition can be adjusted into the following expression:

$$
\begin{aligned}
\dot{V} \leq & -\tilde{x}^{T} U \tilde{x}+2 \Upsilon \lambda_{\max }(W)\|\tilde{x}\|+4 \mu_{2}^{2} \\
& +\mu_{3}^{2} \lambda_{\max }\left(\Pi^{-1} \Pi^{-1}\right) \\
\leq & -\lambda_{\min }(U)\|\tilde{x}\|^{2}+2 \Upsilon \lambda_{\max }(W)\|\tilde{x}\|+\Psi,
\end{aligned}
$$

where $\lambda_{\min }(U)$ denotes the minimum eigenvalue of matrix $U$ which satisfies Theorem $5, \Upsilon=\left(\mu_{0}+\mu_{6}\right)$ and $\Psi=4 \mu_{2}^{2}+$ $\mu_{3}^{2} \lambda_{\max }\left(\Pi^{-1} \Pi^{-1}\right)$, and $\mu_{3}$ has been defined in (6).

Next, in order to complete the stability analysis, two cases will be examined.

Case $1(\|\tilde{x}\|>\eta)$. Consider $\eta=\left(2 \Upsilon \lambda_{\max }(W)+\right.$ $\left.\sqrt{4 \Upsilon^{2} \lambda_{\max }^{2}(W)+4 \Psi \lambda_{\min }(U)}\right) / 2 \lambda_{\text {min }}(U)$.

In this case, $\dot{V}<0$ is established and (8) is a stable observer of nonlinear fault system (2).

Case $2(\|\tilde{x}\| \leq \eta)$. In this case, $\dot{V}>0$ is established and observer (8) will be divergent. This causes the increase of $\|\tilde{x}\|$, and let $\|\tilde{x}\|>\eta$. Next, it will satisfy Case 1 , which brings $\|\tilde{x}\|$ to reduce, and so on; namely, the states error $\|\tilde{x}\|$ will remain at a certain level range. 
Consolidating Cases 1 and 2, it can be seen that the observer is stable, and the designed fault detection observer is able to keep accurate tracking of fault system (2).

Remark 7. $H$ in (14) is a finite gain matrix of fault detection observer (8), which just needs to satisfy the condition that ensures that $(-H C)$ is a stable matrix. For example, the following choice is reasonable:

$$
H=\Theta W C^{T}
$$

where $\Theta>0$ is an adjustable parameter.

\section{Experiments}

Experiment 1. It is a comparative study between the proposed approach and the T-S fuzzy observer.

In order to fully evaluate the performance of the proposed fault diagnosis algorithm, an actuator motion model is selected and its parameters are analyzed as the following expression:

$$
\begin{aligned}
g(x) & =\left[\begin{array}{c}
x_{2} \\
-0.5 \sin \left(x_{1}\right)
\end{array}\right], \\
B & =\left[\begin{array}{ll}
1.5 & 0 \\
0 & 1
\end{array}\right], \\
C & =M=N=\left[\begin{array}{ll}
1 & 0 \\
0 & 1
\end{array}\right] .
\end{aligned}
$$

Obviously, $g(x)$ satisfies the Lipschitz condition. The parameters in fault detection observer (8) are selected according to (14) with the following form:

$$
\begin{aligned}
\delta & =0.5, \\
W & =H=\left[\begin{array}{cc}
5.2 & -4.9 \\
-4.9 & 6.3
\end{array}\right], \\
U & =\left[\begin{array}{cc}
76.08 & -84.53 \\
-84.53 & 95.05
\end{array}\right] .
\end{aligned}
$$

By combining (27) and (28), the fault detection observer based on our proposed algorithm in this paper has been constructed as follows:

$$
\begin{aligned}
{\left[\begin{array}{c}
\dot{\hat{x}}_{1} \\
\dot{\hat{x}}_{2}
\end{array}\right]=} & {\left[\begin{array}{c}
\hat{x}_{2} \\
-0.5 \sin \left(\hat{x}_{1}\right)
\end{array}\right]+\left[\begin{array}{cc}
1.5 & 0 \\
0 & 1
\end{array}\right]\left[\begin{array}{l}
u_{1}+\hat{f}_{1} \\
u_{2}+\hat{f}_{2}
\end{array}\right] } \\
& +\left[\begin{array}{cc}
5.2 & -4.9 \\
-4.9 & 6.3
\end{array}\right]\left[\begin{array}{l}
y_{1}-\hat{y}_{1} \\
y_{2}-\hat{y}_{2}
\end{array}\right], \\
{\left[\begin{array}{l}
\hat{y}_{1} \\
\hat{y}_{2}
\end{array}\right]=} & {\left[\begin{array}{ll}
1 & 0 \\
0 & 1
\end{array}\right]\left[\begin{array}{l}
\hat{x}_{1} \\
\hat{x}_{2}
\end{array}\right] . }
\end{aligned}
$$

The convergence factor matrix $\Pi$ in adaptive fault estimation algorithm (11) is selected carefully as

$$
\Pi=\Pi_{1}=\left[\begin{array}{cc}
1.53 & 0 \\
0 & 6.0
\end{array}\right]
$$

The observer based on Takagi-Sugeno (T-S) fuzzy models is another important approach for fault detection. So it is necessary to make a comparative study between the proposed approach and T-S fuzzy observer. Here, a reduced-order T-S fuzzy observer scheme has been cited and its design process is presented briefly (the specific technical details can be found in [20]).

The T-S fuzzy system fault model that contains actuators' failures is given in the following form:

$$
\begin{aligned}
& \dot{x}=\sum_{i=1}^{l} h_{i}(x)\left(A_{i} x+B_{i}(u+f)\right), \\
& y=C x,
\end{aligned}
$$

where $l$ is the number of fuzzy rules; $h_{i}(x)$ is a membership function and $h_{i}(x) \in[0,1] ; \sum_{i=1}^{l} h_{i}(x)=1 ; A_{i}$ and $B_{i}$ are appropriately dimensional constant matrices; $x, y, u, f$, and $C$ have been defined in (1) and (2) separately.

The fault detection observer based on T-S fuzzy model is designed:

$$
\begin{aligned}
& \dot{\hat{x}}=\sum_{i=1}^{l} h_{i}(\widehat{x})\left(A_{i} \widehat{x}+B_{i}(u+\widehat{f})\right), \\
& \widehat{y}=C \widehat{x},
\end{aligned}
$$

where $\hat{x}, \hat{y}, \hat{f}$, and $C$ have been defined in (8).

A T-S fuzzy adaptive fault estimation algorithm for fault system (2) is given by

$$
\dot{\hat{f}}=\sum_{i=1}^{l} h_{i}(\widehat{x}) \Gamma \tilde{x} .
$$

Assumed in the diagnosis process, there are no disturbances (internal or external); namely, $d=n=[\overline{0}]$, but there are failures described as

$$
f=\left[\begin{array}{l}
0.1 \cos (3.14 \cdot t) \\
0.4 \sin (3.14 \cdot t)
\end{array}\right] \text {. }
$$

The control input vector adopts constants with the following form:

$$
u=\left[\begin{array}{c}
0.02 \\
0.6
\end{array}\right]
$$




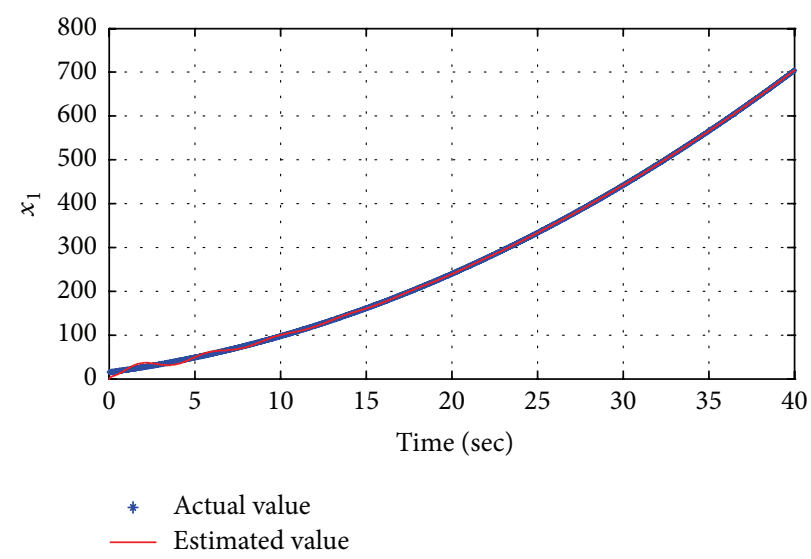

FIGURE 1: Estimation of $x_{1}$ based on the proposed approach.

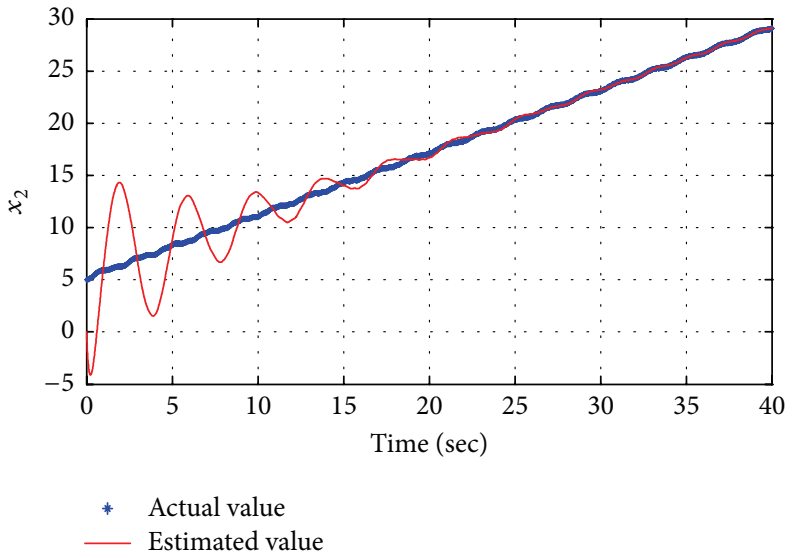

FIGURE 2: Estimation of $x_{2}$ based on the proposed approach.

Initial state vector $x$ and estimation vector $\hat{x}$ are selected as follows:

$$
\begin{aligned}
& x=\left[\begin{array}{c}
15.0 \\
5.0
\end{array}\right], \\
& \widehat{x}=\left[\begin{array}{l}
0.0 \\
0.0
\end{array}\right] .
\end{aligned}
$$

The simulation process lasts 40 seconds. The effects of state estimation and fault estimation based on the proposed algorithm in this paper are shown in Figures 1, 2, 3, and 4. The effects of state estimation and fault estimation based on T-S fuzzy model are shown in Figures 5, 6, 7, and 8.

From Experiment 1, the following can be seen:

(1) There is no obvious difference between the performances of the proposed algorithm in this paper and the T-S fuzzy model. Both of the two algorithms can accurately estimate the state variables and the failures, and only small errors exist. The adjustment time of TS fuzzy model is slightly shorter, but it is not obvious (see Figure 2 versus Figure 6, Figure 3 versus Figure 7, and Figure 4 versus Figure 8). In addition, from the point of view of the design process in two observers, the proposed algorithm is more concise.

(2) The T-S fuzzy observer proposed by [20] did not consider the influence of time-varying disturbances (random measurement noise, external disturbances, etc.) on estimation performance and the solution to the antidisturbance problem was also not given. However, the proposed algorithm in this paper has excellent antidisturbance performance, which is the most important advantage compared with the existing approaches. In order to fully illustrate this point, Experiment 2 is further carried out.

Experiment 2. It is the performance test with the presence of disturbances and failures.

Assuming that in the diagnosis process there are external time-varying disturbances as shown in (37) and time-varying failures as shown in (34) at the same time,

$$
d=\left[\begin{array}{c}
0.08 \cos (31.4 \cdot t) \\
0.3 \sin (31.4 \cdot t)
\end{array}\right]
$$




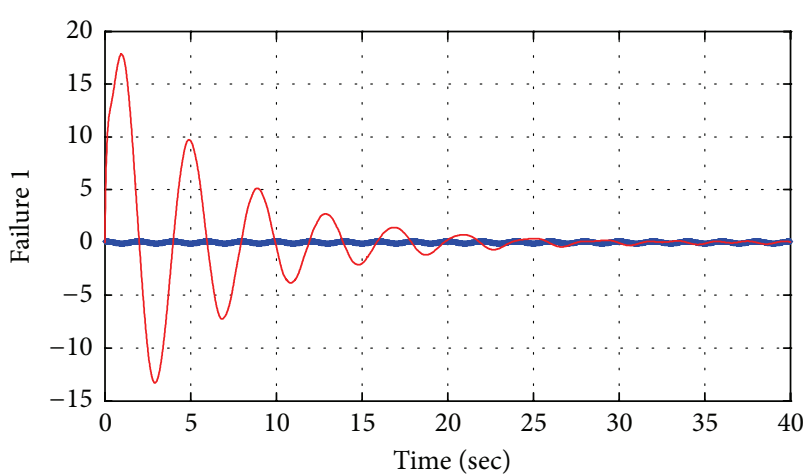

* Actual value

Estimated value

(a)

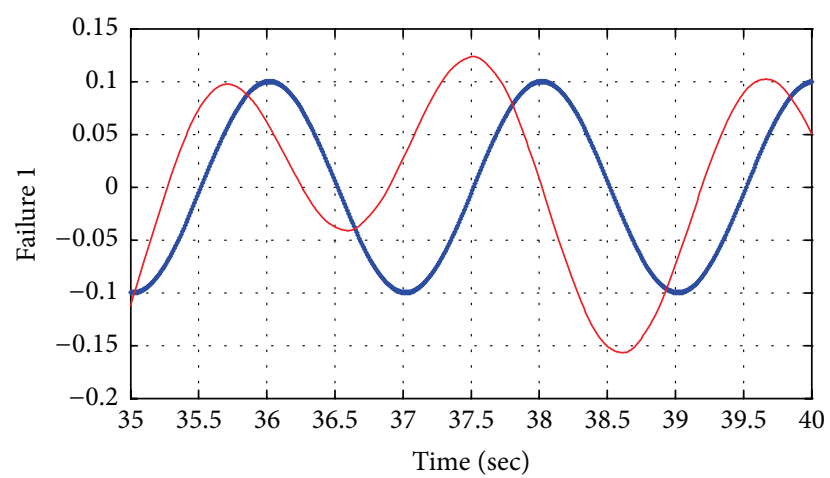

* Actual value

- Estimated value

(b)

FIGURE 3: Estimation of failure 1 based on the proposed approach: (a) estimation of failure 1 and (b) enlarged view.

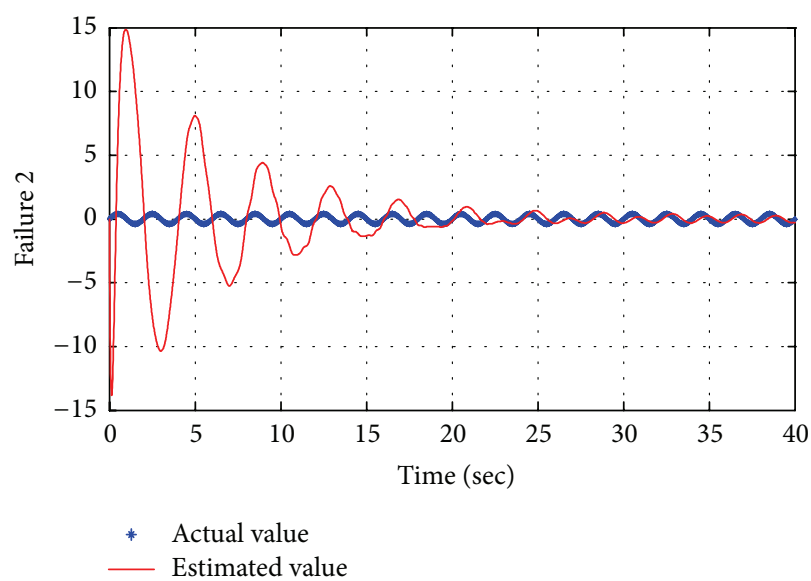

(a)

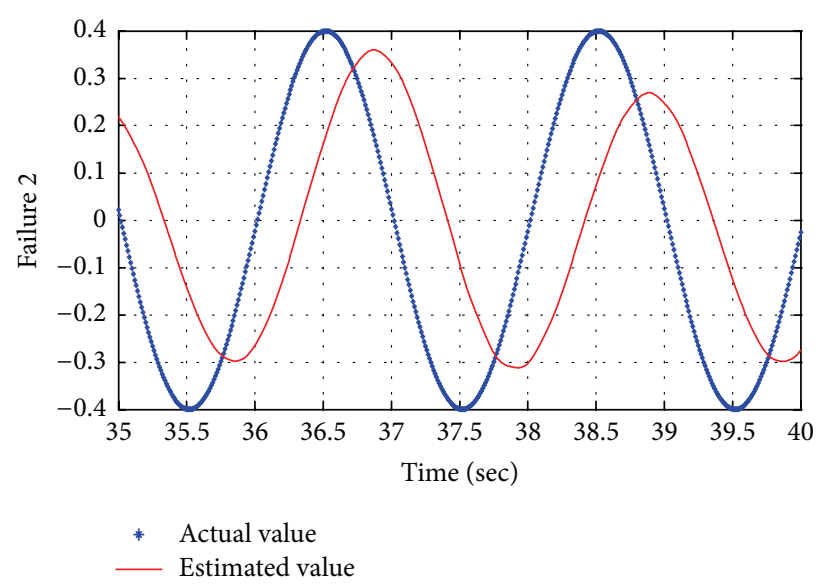

(b)

FIGURE 4: Estimation of failure 2 based on the proposed approach: (a) estimation of failure 2 and (b) enlarged view.

In addition, sensors' random measurement noise $n(t)$ is seen as a band-limited white noise with the height of the power spectral density (PSD) being equal to 0.01 and zero mean, and its variance is equal to 1; namely,

$$
\begin{aligned}
\operatorname{PSD}(n) & =0.01, \\
\operatorname{mean}(n) & =0, \\
\operatorname{var}(n) & =1 .
\end{aligned}
$$

The control input vector also uses (35); initial state vector $x$ and estimation vector $\hat{x}$ use (36).

In order to thoroughly analyze and evaluate the convergence factors' impact on the performance of state estimation and fault estimation, the convergence factor matrix $\Pi$ in adaptive fault estimation algorithm (11) is selected as (30) and (39), respectively:

$$
\Pi=\Pi_{2}=0.5 * \Pi_{1}=\left[\begin{array}{cc}
0.765 & 0 \\
0 & 3.0
\end{array}\right] .
$$

The effects of estimation based on $\Pi_{1}$ are shown in Figures 9-12. The effects of estimation based on $\Pi_{2}$ are shown in Figures 13-16.

For the proposed algorithm in this paper, the following can be concluded from Experiments 1 and 2:

(1) The performance of state estimation is perfect. Regardless of whether there are disturbances, the error signals of state estimation in stable state are very small; namely, $\lim _{t \rightarrow \infty}\|\widetilde{x}\| \approx[\widetilde{0}]$ (see Figures $1,2,9,10,13$, and 14). This shows that the designed fault detection observer (8) is not only stable but also very accurate. The precise state estimation provides the basis for the fault diagnosis of small-amplitude failures.

(2) The external disturbances and random measurement noise have a very obvious influence on the results of fault estimation. From Experiment 2, it can be found that, with the presence of disturbances and noise, the values of fault estimation have obvious "burr" 


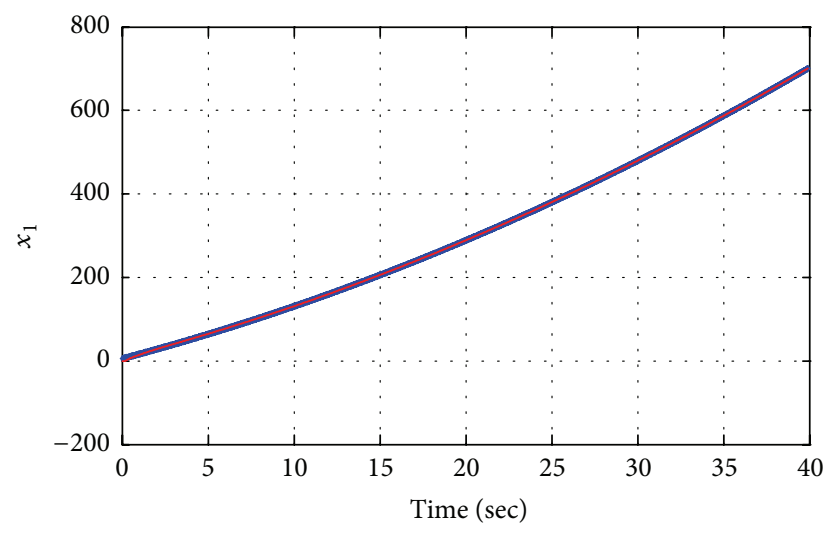

* Actual value

_ Estimated value

FIgURE 5: Estimation of $x_{1}$ based on the T-S fuzzy models.

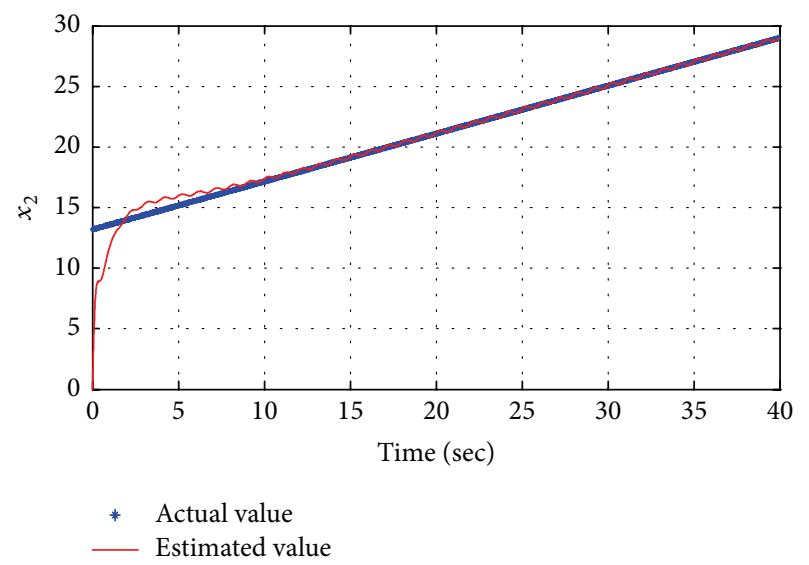

Figure 6: Estimation of $x_{2}$ based on the T-S fuzzy models.

(see Figures 11(b), 12(b), 15(b), and 16(b)). At this time, the estimated failures will oscillate around the actual failures. However, when there are no disturbances or noise, the values of fault estimation are very "smooth" (see Figures 3 and 4). In actual operation, fault system (2) has difficulty avoiding the influence of disturbances and noise, and therefore the results in Experiment 2 are more consistent with the actual situations.

(3) There are slight constant phase differences between actual values and estimated values in steady state (see Figures 3(b), 4(b), 11(b), 12(b), 15(b), and 16(b)). According to Theorem 5, the fault diagnosis algorithm based on fault detection observer (8) and fault estimation algorithm (11) can only ensure that state estimation error $\tilde{x}$ and fault estimation error $\widetilde{f}$ are driven asymptotically to bounded positive constants, not to zero. In other words, the proposed algorithm is not an error-free estimation. So these simulation results are consistent with Lyapunov stability theory and this is why constant phase differences are generated. But the phase differences are not too large and are within an acceptable range.

(4) The convergence factor matrix $\Pi$ has a significant impact on the performance of fault estimation. In Experiment 2, through the comparison of $\Pi_{1}=$ $\left[\begin{array}{cc}1.53 & 0 \\ 0 & 6.0\end{array}\right]$ and $\Pi_{2}=\left[\begin{array}{cc}0.765 & 0 \\ 0 & 3.0\end{array}\right]$, it can be found that if $\Pi_{1}$ is selected, the estimated failures in stable status have smaller amplitude errors (see Figure 11(b) versus Figure 15(b) and Figure 12(b) versus Figure 16(b)). Therefore, an appropriate increase of convergence factors can improve the accuracy of fault estimation and reduce the estimation errors. However, it is also found through experiments that too large values of convergence factors will cause the observer's divergence; therefore, careful selection of convergence factors is always required.

\section{Conclusion}

A nonlinear fault detection observer and an adaptive fault estimation algorithm have been proposed for the diagnosis of a class of nonlinear systems that have actuators' failures. Satisfactory experimental results have been achieved. The most important contribution of this paper is to present a novel diagnostic strategy that is able to give specific estimated values of state variables and failures when the external disturbances and measurement noise exist simultaneously. It has been proved that the fault detection observer, obtained via a Lyapunov function, is useful to achieve asymptotical stability in the case where unknown bounded disturbances and noise exist.

In this paper, we only discuss the problem of detection and estimation of actuators' failures. Future works will be focused on three aspects: the first aspect is the selection of convergence factors $\Pi$ in (11). A guided approach of parameters' selection should be studied to guarantee that the proposed algorithm has faster estimation speed and smaller estimation errors; the second aspect is diagnosis algorithm's spreading. We hope that not only actuators' failures but also sensors' failures can be detected and estimated simultaneously; the third aspect is the practical use. This paper focuses on the theoretical research and how to apply the given results to engineering practice more effectively is also a major task to be completed in the future.

\section{Competing Interests}

The authors declare that there are no competing interests regarding the publication of this paper.

\section{Acknowledgments}

This work was jointly funded by the National Natural Science Foundation of China and the Civil Aviation Administration of China (Grant no. U1433103), the Natural Science Foundation of Tianjin (Grant no. 13JCYBJC39000), and the Basic R\&D Operating Expenses of CAUC with no. 3122014B002 and no. 3122014D022. 


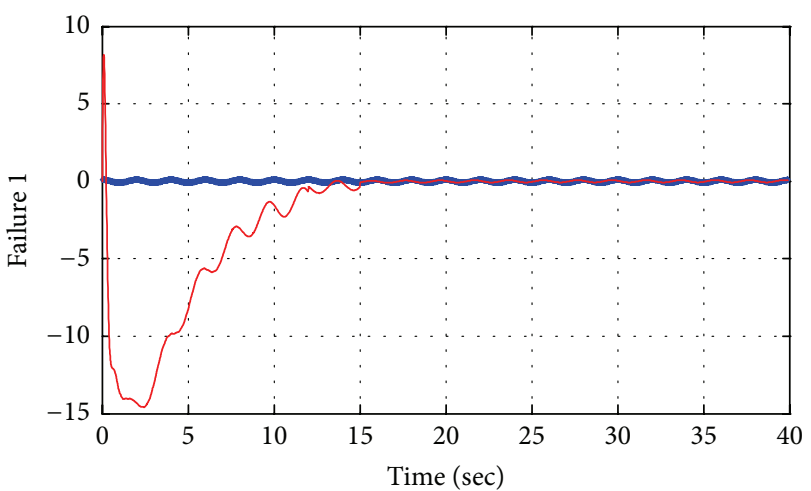

* Actual value

— Estimated value

(a)

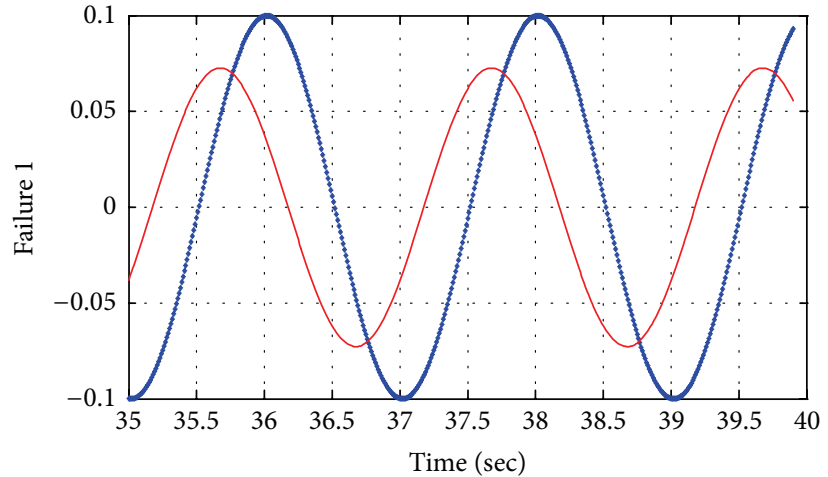

* Actual value

Estimated value

FIGURE 7: Estimation of failure 1 based on the T-S fuzzy models: (a) estimation of failure 1 and (b) enlarged view.

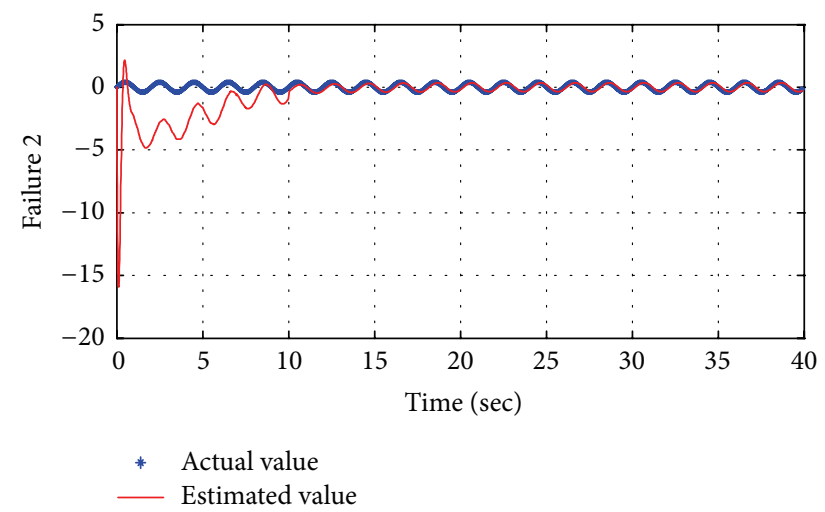

(a)

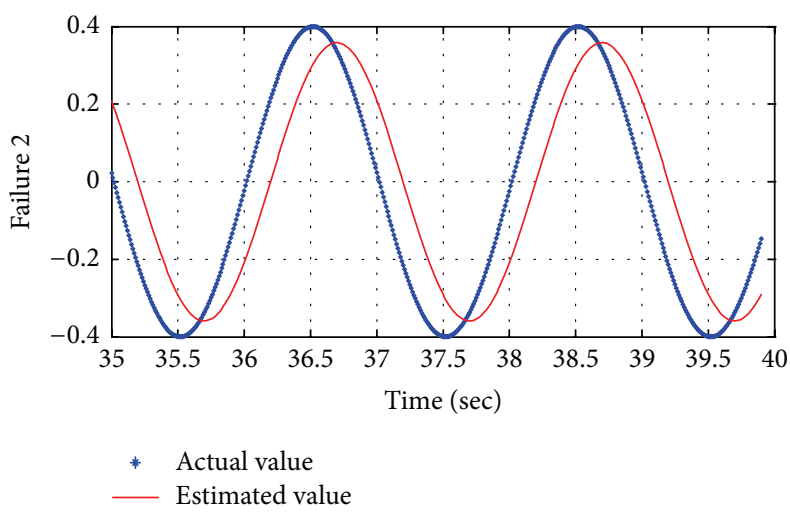

(b)

Figure 8: Estimation of failure 2 based on the T-S fuzzy models: (a) estimation of failure 2 and (b) enlarged view.

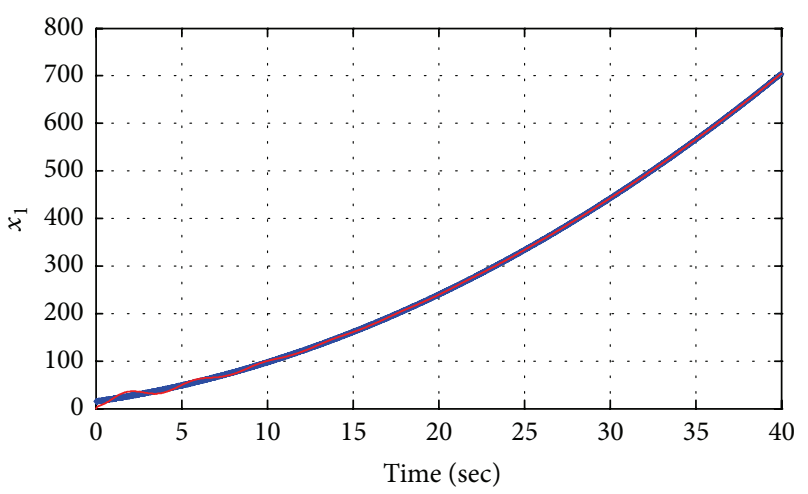

* Actual value

_ Estimated value

FIGURE 9: Estimation of $x_{1}$ with $\Pi_{1}$.

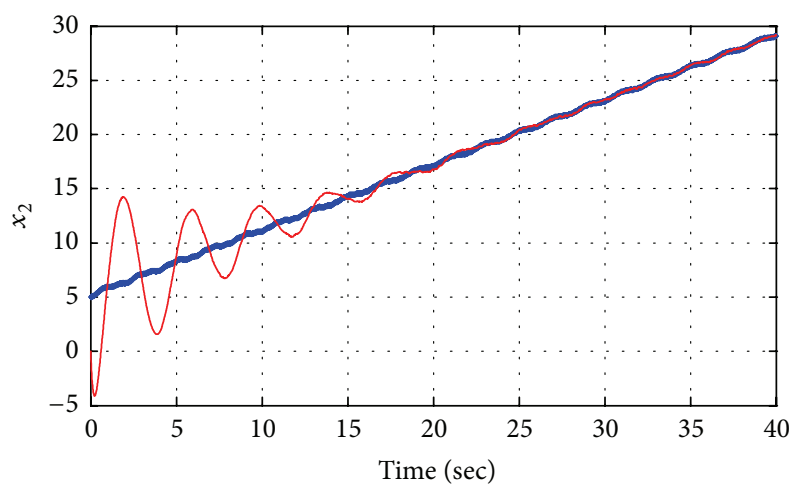

* Actual value

- Estimated value

Figure 10: Estimation of $x_{2}$ with $\Pi_{1}$. 


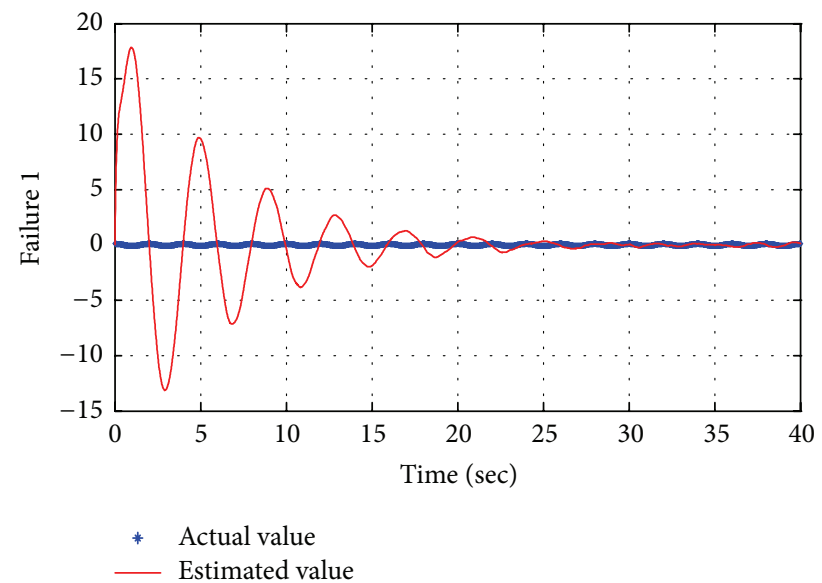

(a)

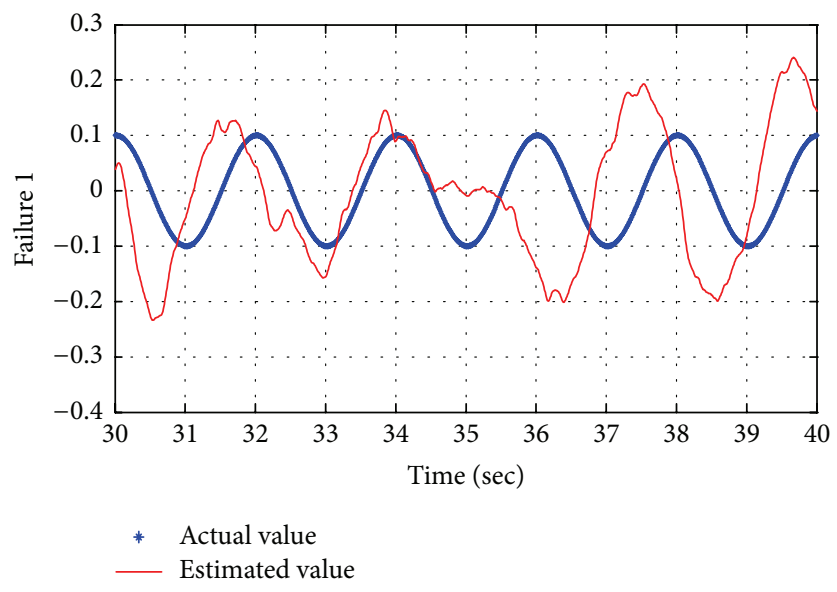

(b)

FIGURE 11: Estimation of failure 1 with $\Pi_{1}$ : (a) estimation of failure 1 and (b) enlarged view.

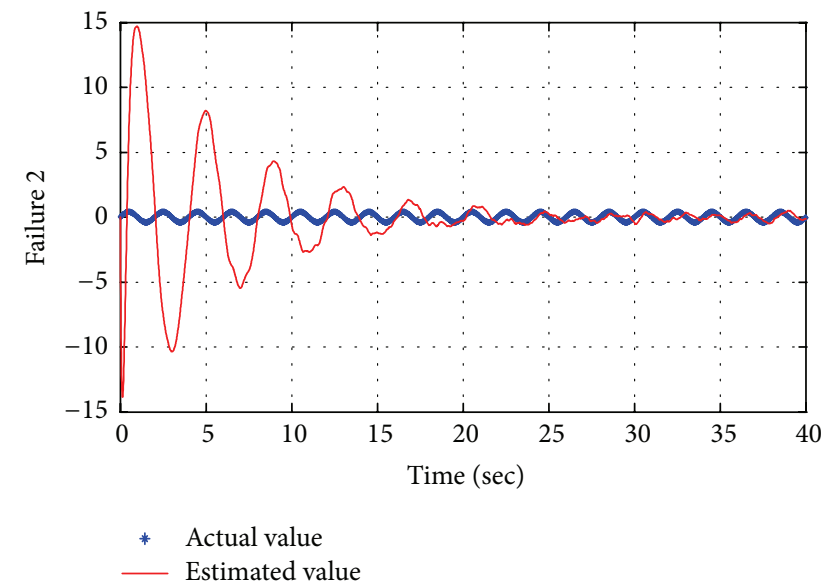

(a)

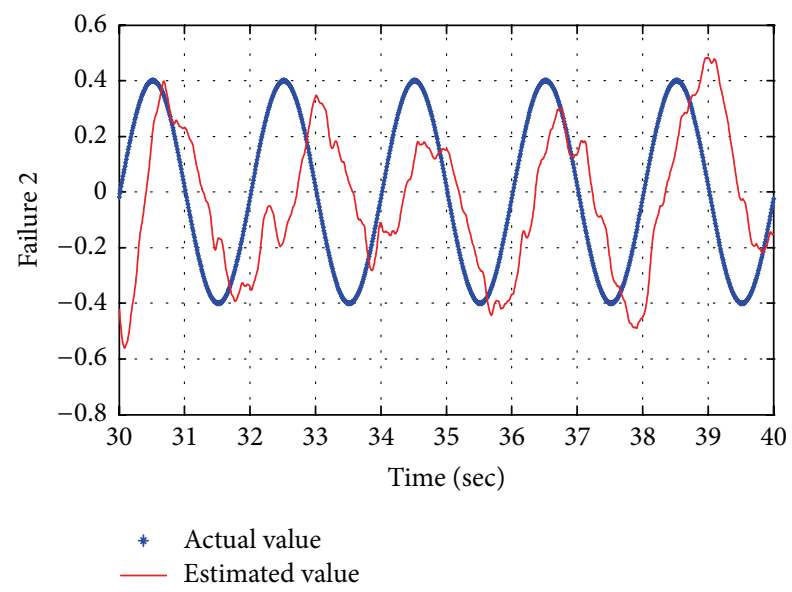

(b)

FIGURE 12: Estimation of failure 2 with $\Pi_{1}$ : (a) estimation of failure 2 and (b) enlarged view.

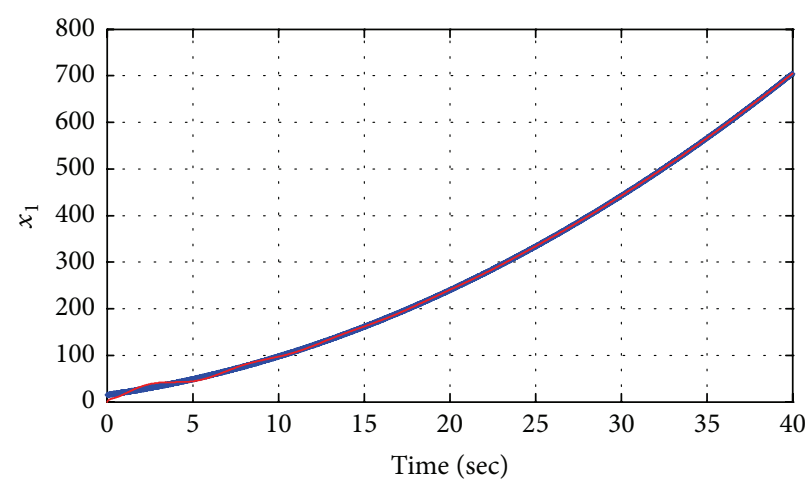

* Actual value

_ Estimated value

FIGURE 13: Estimation of $x_{1}$ with $\Pi_{2}$. 


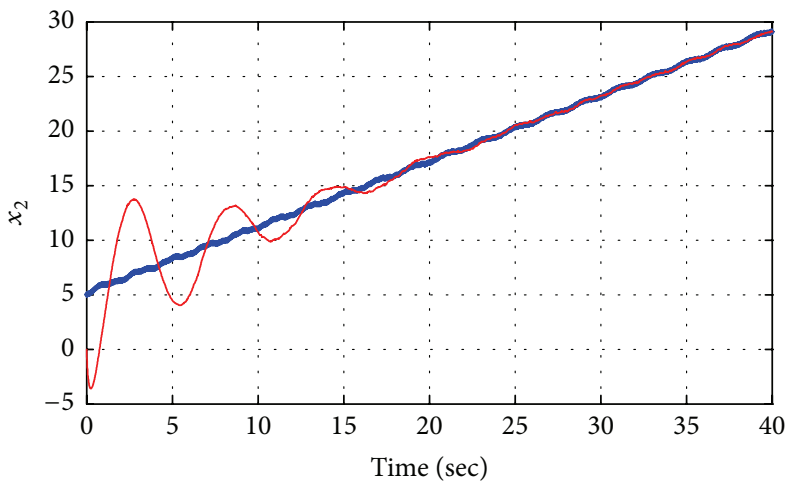

* Actual value

Estimated value

FIgURE 14: Estimation of $x_{2}$ with $\Pi_{2}$.

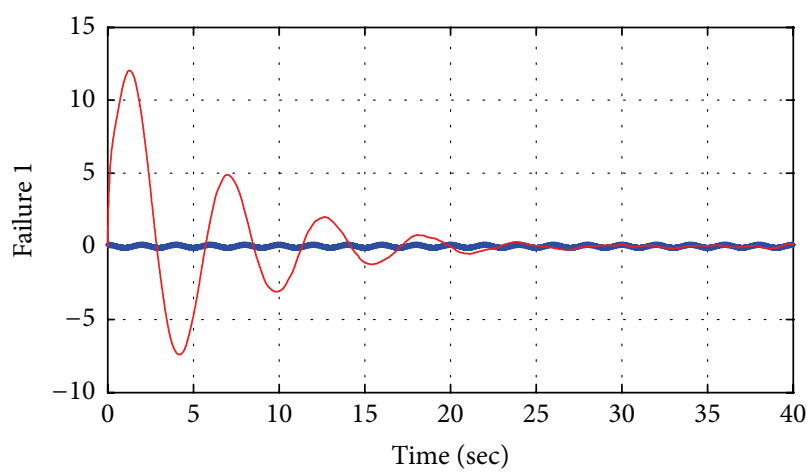

* Actual value

_ Estimated value

(a)

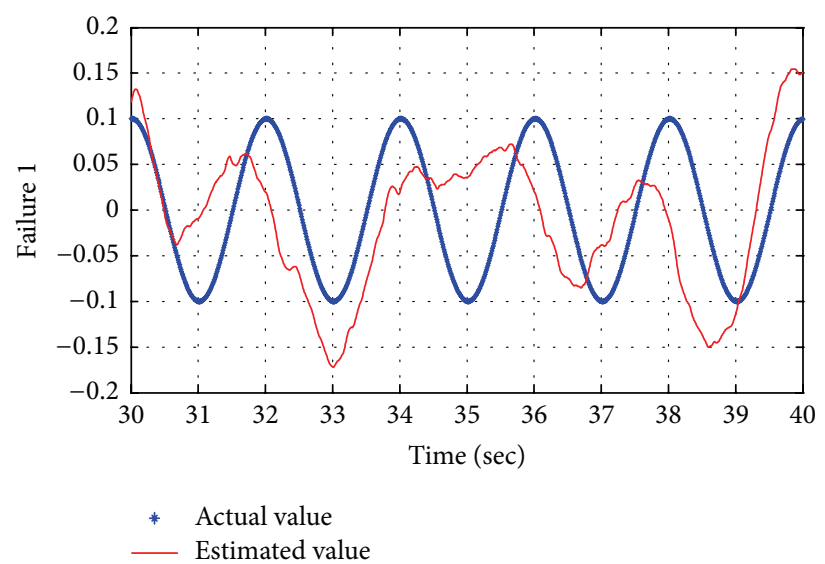

(b)

FIGURE 15: Estimation of failure 1 with $\Pi_{2}$ : (a) estimation of failure 1 and (b) enlarged view.

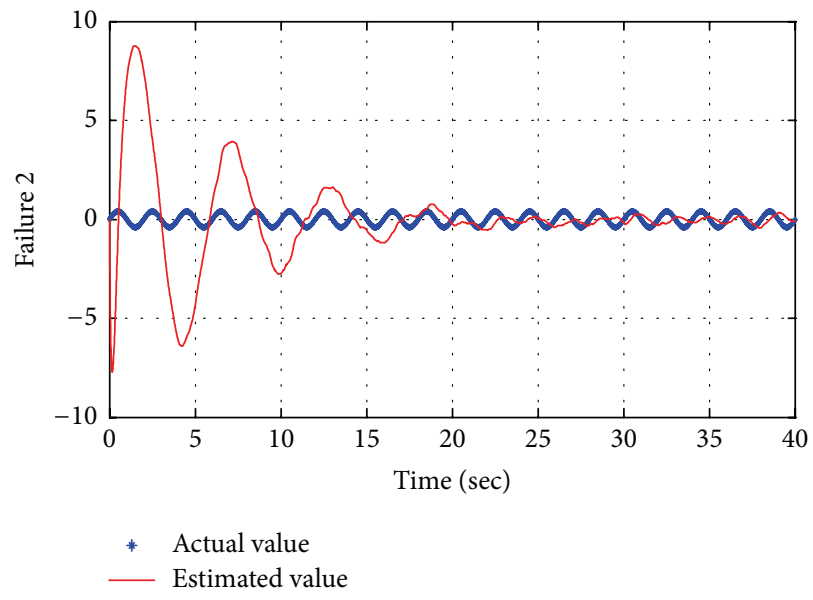

(a)

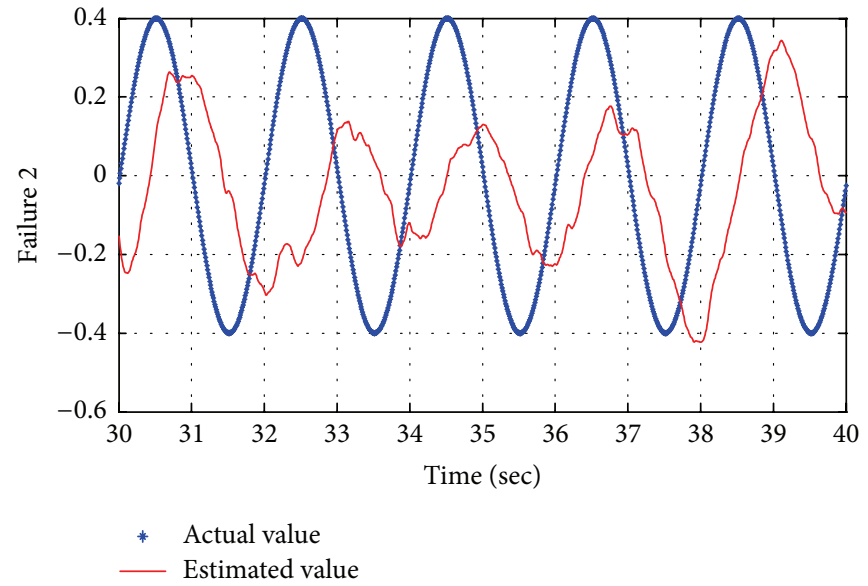

(b)

FIGURE 16: Estimation of failure 2 with $\Pi_{2}$ : (a) estimation of failure 2 and (b) enlarged view. 


\section{References}

[1] M. Chadli, S. Aouaouda, H. R. Karimi, and P. Shi, "Robust fault tolerant tracking controller design for a VTOL aircraft," Journal of the Franklin Institute, vol. 350, no. 9, pp. 2627-2645, 2013.

[2] S. Aouaouda, M. Chadli, M. T. Khadir, and T. Bouarar, "Robust fault tolerant tracking controller design for unknown inputs T-S models with unmeasurable premise variables," Journal of Process Control, vol. 22, no. 5, pp. 861-872, 2012.

[3] S. Aouaouda, M. Chadli, and H.-R. Karimi, "Robust static output-feedback controller design against sensor failure for vehicle dynamics," IET Control Theory and Applications, vol. 8, no. 9, pp. 728-737, 2014.

[4] H. R. Karimi, M. Zapateiro, and N. Luo, "A linear matrix inequality approach to robust fault detection filter design of linear systems with mixed time-varying delays and nonlinear perturbations," Journal of the Franklin Institute, vol. 347, no. 6, pp. 957-973, 2010.

[5] Y. Y. Xu, S. C. Tong, and Y. M. Li, "Prescribed performance fuzzy adaptive fault-tolerant control of non-linear systems with actuator faults," IET Control Theory and Applications, vol. 8, no. 6, pp. 420-431, 2014.

[6] A. Bregon, G. Biswas, B. Pulido, C. Alonso-Gonzalez, and H. Khorasgani, "A common framework for compilation techniques applied to diagnosis of linear dynamic systems," IEEE Transactions on Systems, Man, and Cybernetics: Systems, vol. 44, no. 7, pp. 863-876, 2014.

[7] H. Z. Hosseinabadi, B. Nazari, R. Amirfattahi, H. R. Mirdamadi, and A. R. Sadri, "Wavelet network approach for structural damage identification using guided ultrasonic waves," IEEE Transactions on Instrumentation and Measurement, vol. 63, no. 7, pp. 1680-1692, 2014

[8] J. Li and G.-H. Yang, "Fault detection filter design for discretetime switched linear systems with mode-dependent average dwell-time," International Journal of Adaptive Control and Signal Processing, vol. 28, no. 1, pp. 77-95, 2014.

[9] S. Yin and X. Zhu, "Intelligent particle filter and its application to fault detection of nonlinear system," IEEE Transactions on Industrial Electronics, vol. 62, no. 6, pp. 3852-3861, 2015.

[10] L.-Y. Meng and B. Jiang, "Fault diagnosis based on the nonlinear adaptive observer," Systems Engineering and Electronics, vol. 30, no. 7, pp. 1317-1319, 2008.

[11] S. Kelkar and R. Kamal, "Adaptive fault diagnosis algorithm for controller area network," IEEE Transactions on Industrial Electronics, vol. 61, no. 10, pp. 5527-5537, 2014.

[12] S. Aouaouda, M. Chadli, P. Shi, and H. R. Karimi, "Discretetime $\mathrm{H}-/ \mathrm{H} \infty$ sensor fault detection observer design for nonlinear systems with parameter uncertainty," International Journal of Robust and Nonlinear Control, vol. 25, no. 3, pp. 339-361, 2015.

[13] S. Aouaouda, M. Chadli, V. Cocquempot, and K. M. Tarek, "Multi-objective $H_{-} / H_{\infty}$ fault detection observer design for Takagi-Sugeno fuzzy systems with unmeasurable premise variables: descriptor approach," International Journal of Adaptive Control and Signal Processing, vol. 27, no. 12, pp. 1031-1047, 2013.

[14] M. Chadli, A. Abdo, and S. X. Ding, " $H_{-} / H_{\infty}$ fault detection filter design for discrete-time Takagi-Sugeno fuzzy system," Automatica, vol. 49, no. 7, pp. 1996-2005, 2013.

[15] E. Alcorta-García, A. Zolghadri, and P. Goupil, "A nonlinear observer-based strategy for aircraft oscillatory failure detection: A380 case study," IEEE Transactions on Aerospace and Electronic Systems, vol. 47, no. 4, pp. 2792-2806, 2011.
[16] Y. Han, S. Oh, B. Choi, D. Kwak, H. J. Kim, and Y. Kim, "Fault detection and identification of aircraft control surface using adaptive observer and input bias estimator," IET Control Theory and Applications, vol. 6, no. 10, pp. 1367-1387, 2012.

[17] M. Rodrigues, H. Hamdi, D. Theilliol, C. Mechmeche, and N. Benhadj Braiek, "Actuator fault estimation based adaptive polytopic observer for a class of LPV descriptor systems," International Journal of Robust and Nonlinear Control, vol. 25, no. 5, pp. 673-688, 2015.

[18] J. Zhang, A. K. Swain, and S. K. Nguang, "Robust Ho adaptive descriptor observer design for fault estimation of uncertain nonlinear systems," Journal of the Franklin Institute, vol. 351, no. 11, pp. 5162-5181, 2014.

[19] R. X. Guo, K. Guo, J. K. Dong, and Y. Zhu, "Time-varying and anti-disturbance fault diagnosis for a class of nonlinear systems," Proceedings of the Institution of Mechanical Engineers, Part I: Journal of Systems and Control Engineering, vol. 229, no. 7, pp. 573-586, 2015.

[20] D. Krokavec and A. Filasová, "A reduced-order TS fuzzy observer scheme with application to actuator faults reconstruction," Mathematical Problems in Engineering, vol. 2012, Article ID 951953, 25 pages, 2012. 


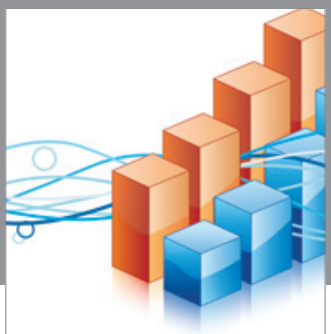

Advances in

Operations Research

vatem alat4

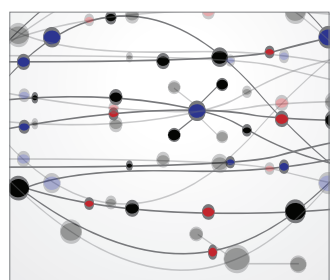

\section{The Scientific} World Journal
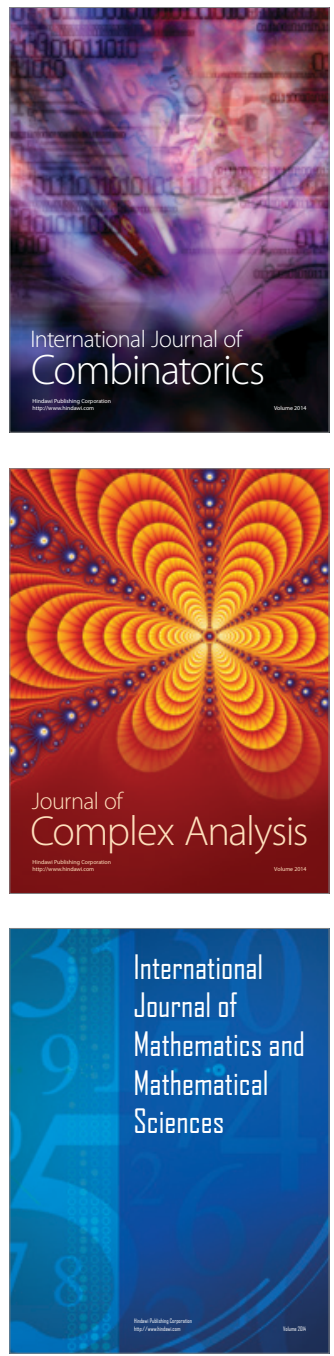
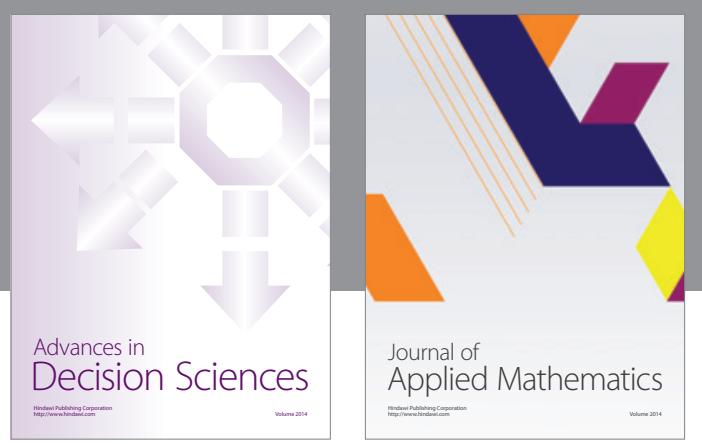

Algebra

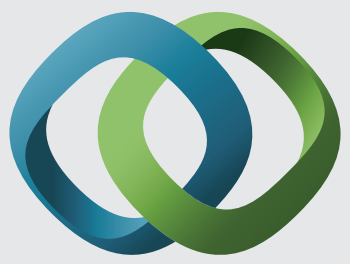

\section{Hindawi}

Submit your manuscripts at

http://www.hindawi.com
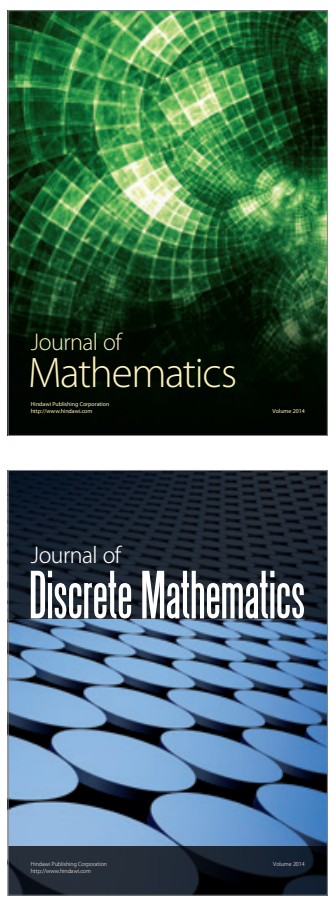

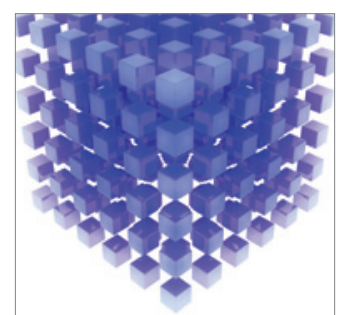

Mathematical Problems in Engineering
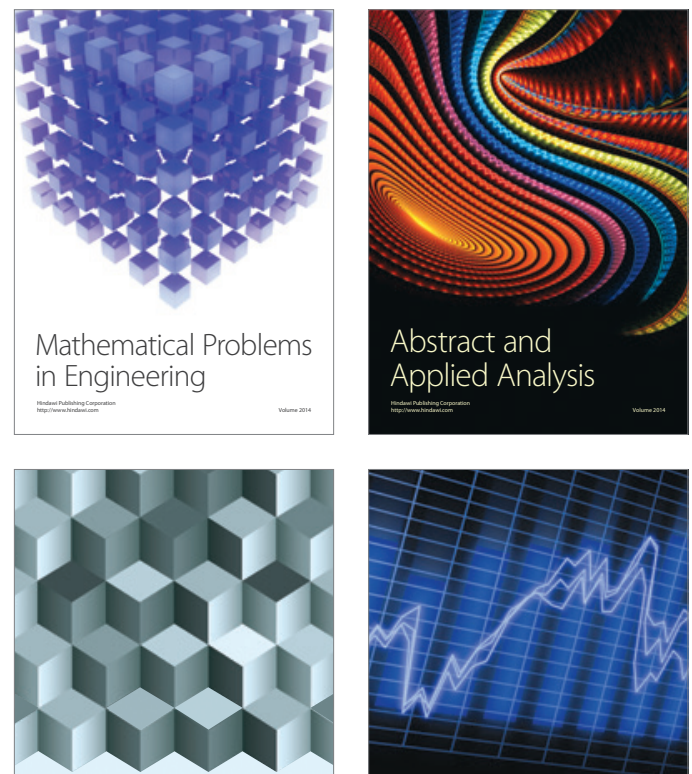

Journal of

Function Spaces

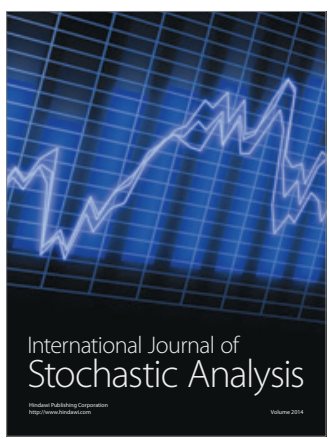

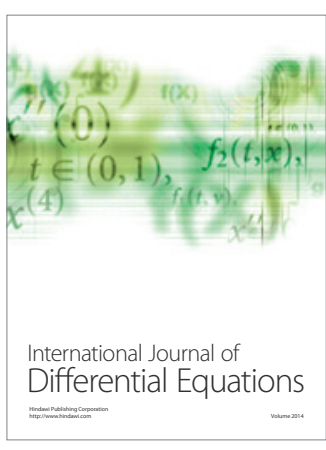
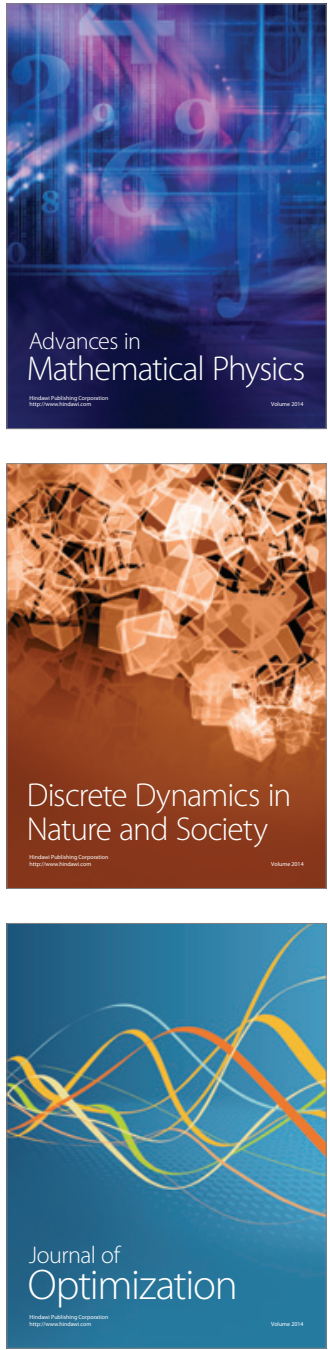